Ann. Sci. forest., I976, 33 (I), 27-45.

\title{
OBSERVATIONS SUR L'ACTIVITÉ DE LA NITRATE RÉDUCTASE \\ ET SUR L'APTITUDE A RÉDUIRE LES NITRITES PAR VOIE NON ENZYMATIQUE CHEZ DES RÉSINEUX CALCICOLES ET CALCIFUGES
}

\author{
A. CLÉMENT et F. LE TACON \\ avec la collaboration technique de Maryse Bitsch \\ Station de Recherches sur les Sols forestiers et la Fertilisation, \\ Centre national de Recherches forestières, I. N.R. A., \\ Champenoux, 54280 Seichamps
}

\section{RÉSUMÉ}

L'activité de la nitrate réductase dans les extraits de tissus résineux est élevée à pH voisin de 7 et faible à pH 3,5. Elle est beaucoup plus élevée dans les racines que dans les aiguilles. 11 existe un temps de latence de plusieurs heures ( 7 à $\mathrm{IO}$ h) avant que l'activité de la nitrate réductase ne démarre. Ce temps de latence est fonction du degré de broyage des tissus et semble dû à la présence d'un inhibiteur enzymatique.

La réduction des nitrites semble se faire par voie non enzymatique. Cette aptitude des tissus à réduire les nitrites est élevée à $\mathrm{pH} 3,5$ et nulle à $\mathrm{pH}$ 7,2. Elle semble plus élevée pour les tissus foliaires que pour les tissus racinaires.

Nous avons comparé ce système de réduction des nitates et des nitrites chez l'Épicéa commun (Picea excelsa) et chez le Pin noir d'Autriche (Pinus nigra nigricans austriaca) en sol acide et en sol calcaire.

Dans les tissus racinaires de Pin noir, l'activité de la nitrate réductase est plus élevée en sol calcaire qu'en sol acide. Nous observons l'inverse pour les tissus racinaires d'Épicéa. Ces observations semblent pouvoir expliquer en partie les difficultés de nutrition en azote que présente l'Épicéa en sol calcaire.

\section{I. - INTRODUCTION}

Un grand nombre de résineux présentent une chlorose généralisée en sol calcaire. Cette chlorose s'accompagne toujours d'une faible croissance et d'une carence très marquée en azote.

Il semble que ces résineux utilisent avec difficulté l'azote nitrique qui est la forme dominante d'azote en sol calcaire. 
Pour expliquer ces difficultés d'utilisation de l'azote nitrique, on peut avancer diverses hypothèses, et en particulier la difficulté de réduire les nitrates.

Nous avons donc été amenés à mesurer l'activité de la nitrate réductase et la capacité à réduire les nitrites chez l'Épicéa, espèce calcifuge, et le Pin noir d'Autriche, espèce résistante au calcaire.

\section{2. - RAPPEI, SUR LES CONNAISSANCES ACTUELLES DU MÉCANISME DE RÉDUCTION DES NITRATES}

On admet que la réduction des nitrates se fait suivant le schéma ci-dessous hypothétique dans ses dernières phases :

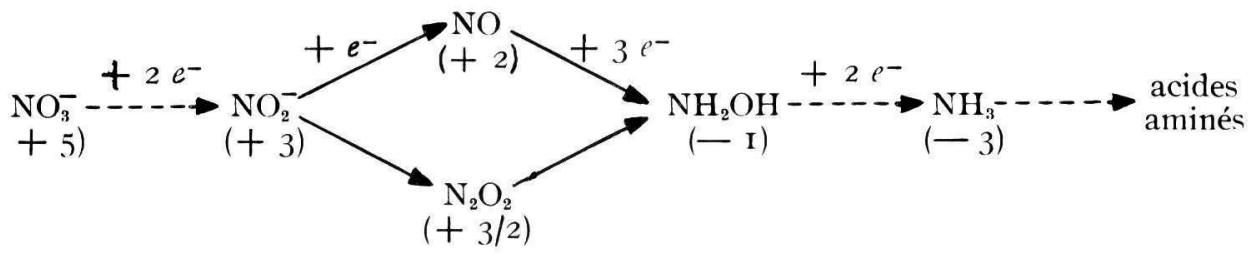

Il semble d'ailleurs, d'après HEwitT et al., que l'hydroxylamine $\mathrm{NH}_{2} \mathrm{OH}$ ne soit pas un intermédiaire indispensable.

Cette réduction se fait par l'intermédiaire d'une chaîne d'enzymes, dont on ne connaît avec certitude que les deux premières.

La nitrate réductase est bien connue. Il s'agit d'une molybdo-flavoprotéine, de poids moléculaire compris entre 500 o00 et 600 o00, et ayant une activité optimale à $\mathrm{pH}$ compris entre 7 et 7,5 .

La nitrite réductase qui fait suite à la nitrate réductase est par contre moins connue. Le fer interviendrait dans son fonctionnement. I)'autres oligo-éléments, comme le manganèse et le cuivre pourraient intervenir également.

La réduction des nitrates a surtout été étudiée chez les bactéries, les champignons et les algues. Un certain nombre d'études ont été effectuées sur des plantes de culture (soja, dactyle, ray-grass, maïs, blé, tomate) et sur des arbres (en particulier sur oranger et citronnier). Citons Bar-Akiva, J. SAgIv, J. Leshem (I970), BarAkiva et Sternbaum (ig65). Sur arbres forestiers, citons les études de W. F. Theobald et W. H. Smith(I974) et de G. F. Dykstra (I974).

\section{3. - MATÉRIEL E'T MÉTHODES}

L'activité de la nitrate réductase peut être mesurée par deux méthodes différentes : la méthode de l'extrait enzymatique ou encore méthode in vitro et la méthode des fragments ou méthode in vivo. Mieux vaut d'ailleurs ne pas utiliser les termes de in vivo ou in vitro; on ne peut en effet assimiler la méthode des fragments à une véritable méthode in vivo. 


\section{1. - La méthode des extraits}

Cette méthode consiste à extraire l'enzyme puis à la concentrer par des purifications successives. L'enzyme est ensuite mise en contact avec des nitrates, un tampon et un donneur d'hydrogène dans des conditions parfaitement contrôlées. Cette méthode a été utilisée tout d'abord en 1953 par Evaxs et Nason, et ensuite par des nombreux autres auteurs.

\section{2. - La méthode des fragments}

Des fragments de matériel végétal de 2 à $3 \mathrm{~mm}$ sont mis en présence d’un tampon phosphate, d'un donneur d'hydrogène (acide malique ou citrique) et de nitrates. Mulder, Boxma et VAx I'Eex (1959), puis Bar-Akiva (I965-I968), Blondel (I97I) ont utilisé cette technique. D'autres auteurs utilisent un tampon Tris (HAgeman et Flesher, Champigny) ; d'autres encore utilisent un tampon pyrophosphate et, comme donneur d'électron, le NADH.

Le phosphate joue un rôle important dans l'activité de l'enzyme en intervenant dans le transport des électrons (Nicholas, Scawin, I956; Blondel, I97I).

Cette méthode est intéressante par sa simplicité et sa reproductibilité, et donnerait peut-être une meilleure représentation de la réalité que la méthode de l'extrait enzymatique.

\section{3. - Méthode utilisée}

Nous avons nous-même utilisé cette méthode des fragments. Les fragments d'aiguilles ou de racines $\left(2 \mathrm{~mm}\right.$ environ) sont mis en incubation à l'obscurité à $28^{\circ} \mathrm{C}$ avec un tampon citrate phosphate proche de celui utilisé par Mulder, Boxma et Van Veen. Le donneur d'électron est l'acide citrique. Les nitrates sont introduits en quantité connue, dès la mise en incubation.

Après incubation, dont la durée est variable suivant les cas, puis une filtration, nous dosons sur le filtrat les nitrites apparus au cours de l'incubation. Le dosage des nitrites est effectué suivant la méthode de BARnes et FolKhard (colorimétrie en présence de dichlorure de naphtyléthylène diamine et de sulfanilamide). La quantité de nitrites apparus dans le milieu d'incubation traduit l'activité de la nitrate réductase.

La mesure du pouvoir de réduction des nitrites a été effectuée suivant les mêmes principes. Nous mettons une quantité connue de nitrites en présence de fragments de tissus et du même tampon citrate-phosphate. Après incubation, puis filtration, nous dosons la quantité de nitrites restant dans le milieu d'incubation, toujours suivant la méthode de Barnes et Folkhard. Par différence, nous obtenons la quantité de nitrites ayant disparu en présence des fragments de tissus. Cette quantité exprime le pouvoir de réduction des nitrites par les tissus.

\section{4. - Échantillonnage}

Nous avons travaillé principalement sur deux espèces insensibles à la chlorose en milieu calcaire (Pinus nigra nigricans et Pinus nigra laricio corsicana) et sur une espèce sensible à la chlorose (Picea excelsa), sur arbres adultes et sur jeunes plants élevés en pépinière, sur aiguilles de l'année et racines.

Les plants issus de pépinière ont tous été élevés sur un substrat identique. Ces jeunes plants ont seuls été utilisés pour la première partie de l'étude (Chapitre IV et V).

Par contre, les arbres adultes proviennent de milieux différents (sol acide et sol calcaire). Seuls ces arbres adultes ont été utilisés pour la deuxième partie de l'étude (Chapitre VI).

Tous les prélèvements ont été effectués en pleine période de végétation, entre le I 5 juillet 1973 et le $I_{5}$ août $I_{973}$, le matin avant Io heures, au moment où l'activité est maximale. Les dosages ont été effectués immédiatement après les prélèvements ou après conservation des échantillons à $0^{\circ} \mathrm{C}$ en glacière pendant le temps des transports.

\section{5. - Choix du pH des tampons}

L'étucle de l'activité de la nitrate réductase est faite généralement entre $\mathrm{pH} 7$ et 7,5, c'est-àdire à l'optimum de l'activité de l'enzyme.

11 nous a semblé intéressant d'étudier l'activité de la nitrate réductase au pH des tissus foliaires ou racinaires des résineux. 
L'un d'entre nous (CLÉMENT, I972) a en effet montré que le pH des broyats de tissus foliaires frais de résineux est compris entre 3,2 et 3,5 .

Nous avons repris des mesures du $\mathrm{pH}$ des tissus foliaires et racinaires de quelques espèces

\begin{tabular}{|c|c|c|}
\hline & $\mathrm{pH}$ foliaire & $\mathrm{pH}$ racinaire \\
\hline Picea excelsa ................. & 3,2 & 5,0 \\
\hline Pinus nigra laricio (corsicana) .... & $3,-2$ & 5,0 \\
\hline Larix europea ................ & 3,1 & 5,0 \\
\hline Populus I $214 \ldots \ldots \ldots \ldots \ldots \ldots$ & 5,8 & 6,1 \\
\hline
\end{tabular}

Nous avons donc retenu $3 \mathrm{pH}$ pour le tampon citrate-phosphate $3,5-5,0$ et 7,2 .

\section{4. - APTITUDE DES TISSUS A RÉDUIRE IES NITRI'TES PAR VOIE NON ENZYMATIQUE}

Lorsque l'on dose la quantité de nitrites apparus dans un milieu d'incubation, on détermine la résultante de deux phénomènes : 1'activité de la nitrate réductase qui produit des nitrites à partir des nitrates, l'activité de la nitrite réductase qui provoque par réduction la disparition des nitrites, ou l'aptitude à réduire directement les nitrites lorsque cette réduction ne se fait pas par voie enzymatique.

A priori, on ne peut donc pas être certain de mesurer l'activité de la seule nitrate réductase en dosant les nitrites apparaissant dans le milieu d'extraction. C'est pour cette raison que nous avons tout d'abord essayé de préciser les conditions de réduction des nitrites, de façon à pouvoir éventuellement éliminer cette influence.

Nous avons conservé le principe de la méthode des fragments, telle qu'elle avait été définie pour mesurer l'activité de la nitrate réductase. Nous introduisons une quantité connue de nitrites dans le milieu contenant le tampon et les fragments de tissus. La capacité de réduction des nitrites est déterminée par mesures périodiques de la quantité de nitrites restants. Cette technique est utilisable dans le cas des résineux car il n'y a au préalable ni nitrates, ni nitrites dans leurs tissus, ou tout au plus quelques traces. Nous avons introduit dans le milieu roo $\mu \mathrm{g}$ et $200 \mu \mathrm{g}$ de nitrites pour ro $\mathrm{g}$ de matériel frais (racines et feuilles de Pin laricio de Corse) et utilisé 3 tampons à $\mathrm{pH} 3,5, \mathrm{pH}, 5, \mathrm{pH} 7,2$.

\section{Caractère non enzymatique de la réaction}

Après ébullition, nous constatons qu'il n'y a aucune diminution du pouvoir de réduction du milieu d'incubation vis-à-vis des nitrites. On ne peut donc attribuer un caractère enzymatique à cette réduction. Les figures I et 2 mettent en évidence que, chez le Pin laricio, la réduction des nitrites est faible à $\mathrm{pH} 7,2$ et très élevée à pH 3,5. D'autre part, la réduction est un peu plus forte dans les feuilles que dans les racines. Nous avons également observé que, chez 1'Épicéa, la réduction des nitrites était quasiment nulle à $\mathrm{pH} 7,2$. 


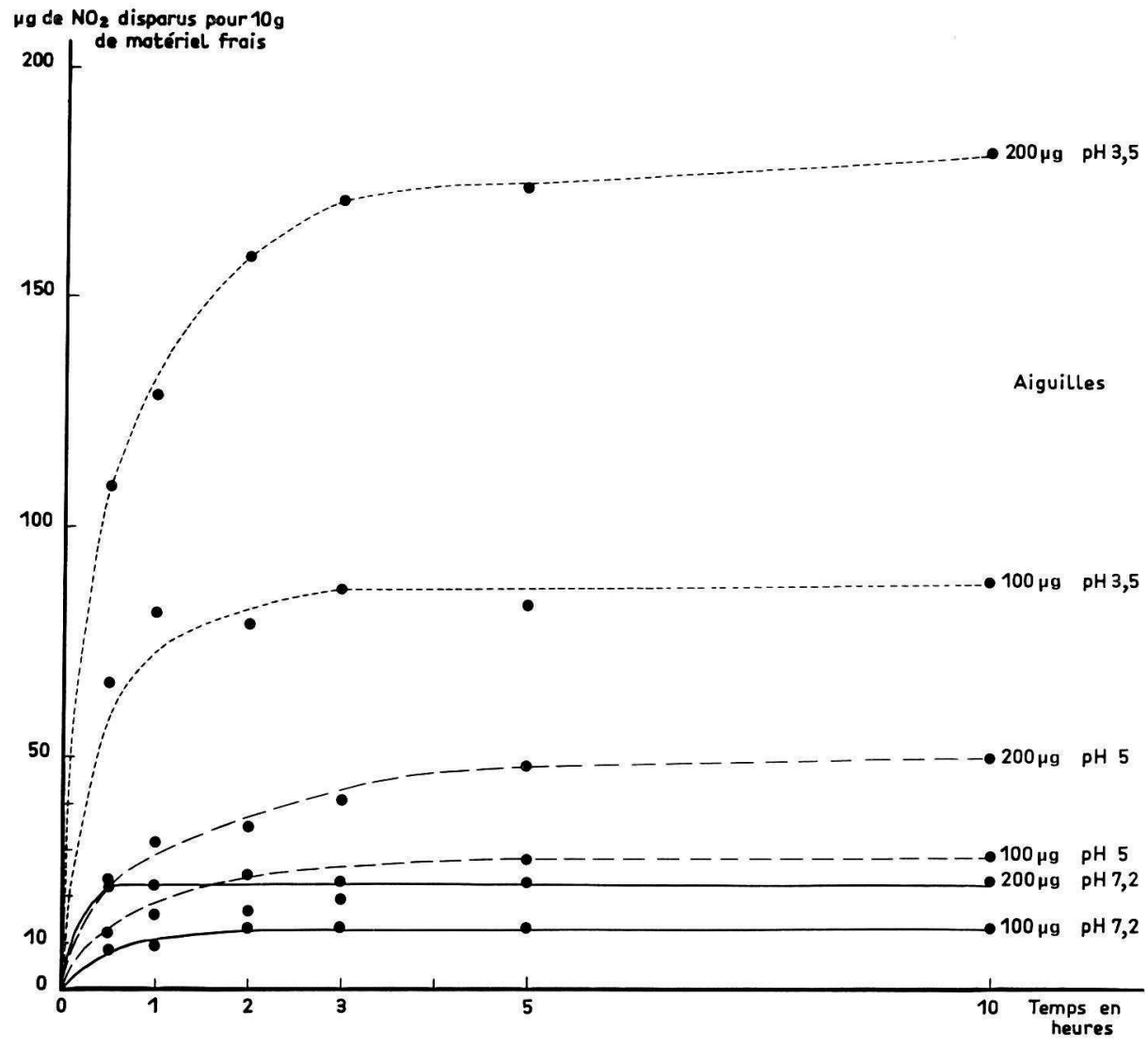

I.r., 1. - Réduction des nitrites par voie non enzymatiqui dans les aiguilles de l'année à $28^{\circ} \mathrm{C}$ (août 1973).

Pinus nigra laricio (corsicana), 2 ans

Nitrite reduction without enzyme system in needle extracts at $: 8^{\circ} \mathrm{C}$.

? years old, Pinus nigra laricio (corsicana) 


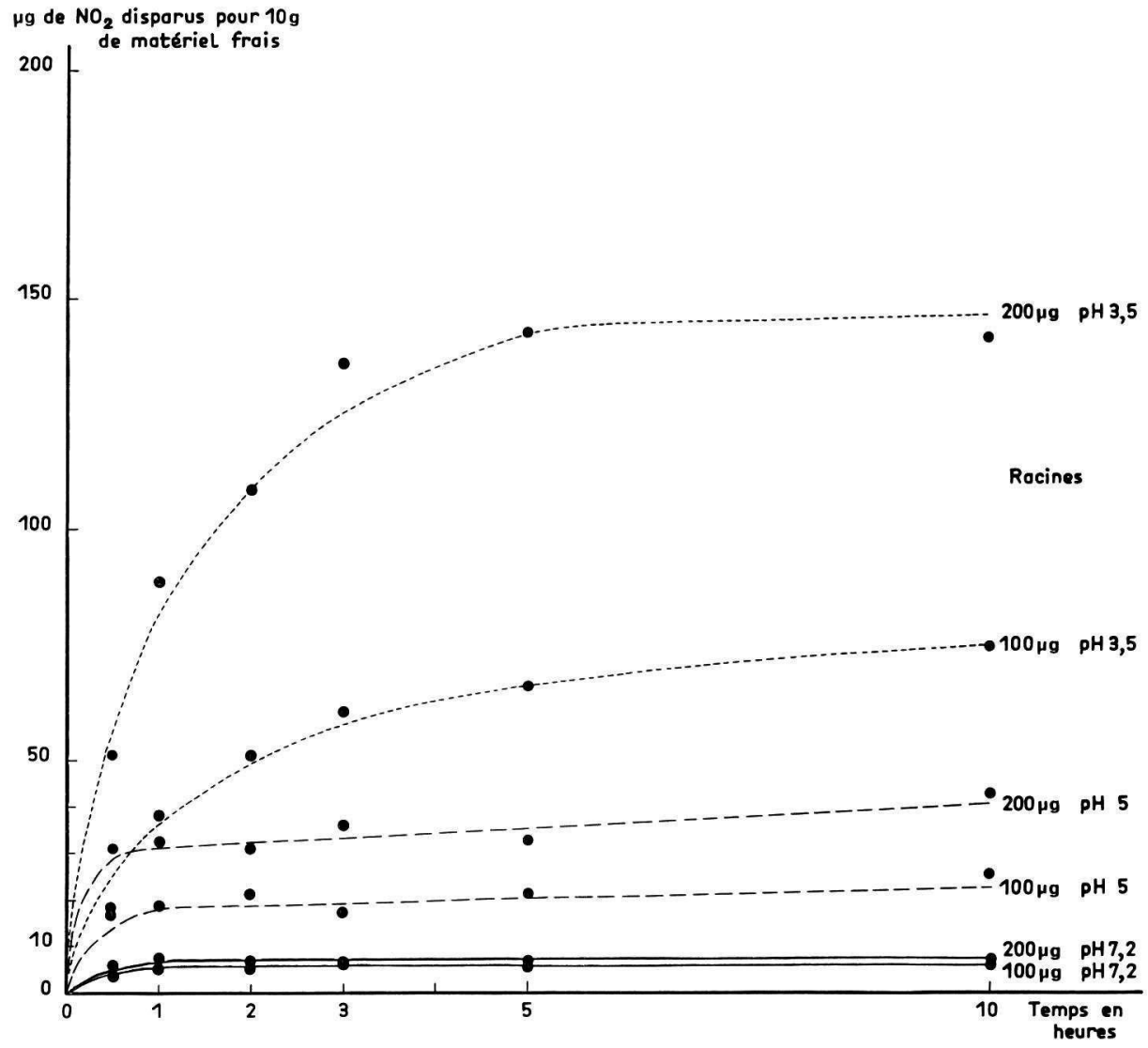

Fir. 2. Réduction des nitrites par voie non enzymatique dans les racines à :280 (août 1!r:3).

Pinus nigra laricio (corsicana), 2 ans

Vitrite reduction without enzyme system in root extracts at $28^{\circ} \mathrm{C}$.

$\because$ years old, Pinus nigra laricio (corsicana) 
On constate également que cette réduction devient nulle au bout de 3 à 4 heures environ, dans tous les cas. Elle peut devenir nulle pour 2 raisons : Il peut ne plus y avoir de nitrites à réduire dans le milieu (cas des fragments de feuilles à $\mathrm{pH} 3,5$ ), ou bien la capacité de réduction des nitrites cesse, bien qu'il y ait encore des nitrites dans le milieu (racines dans tous les cas, feuilles à $\mathrm{pH}_{5}$ et 7,2 ).

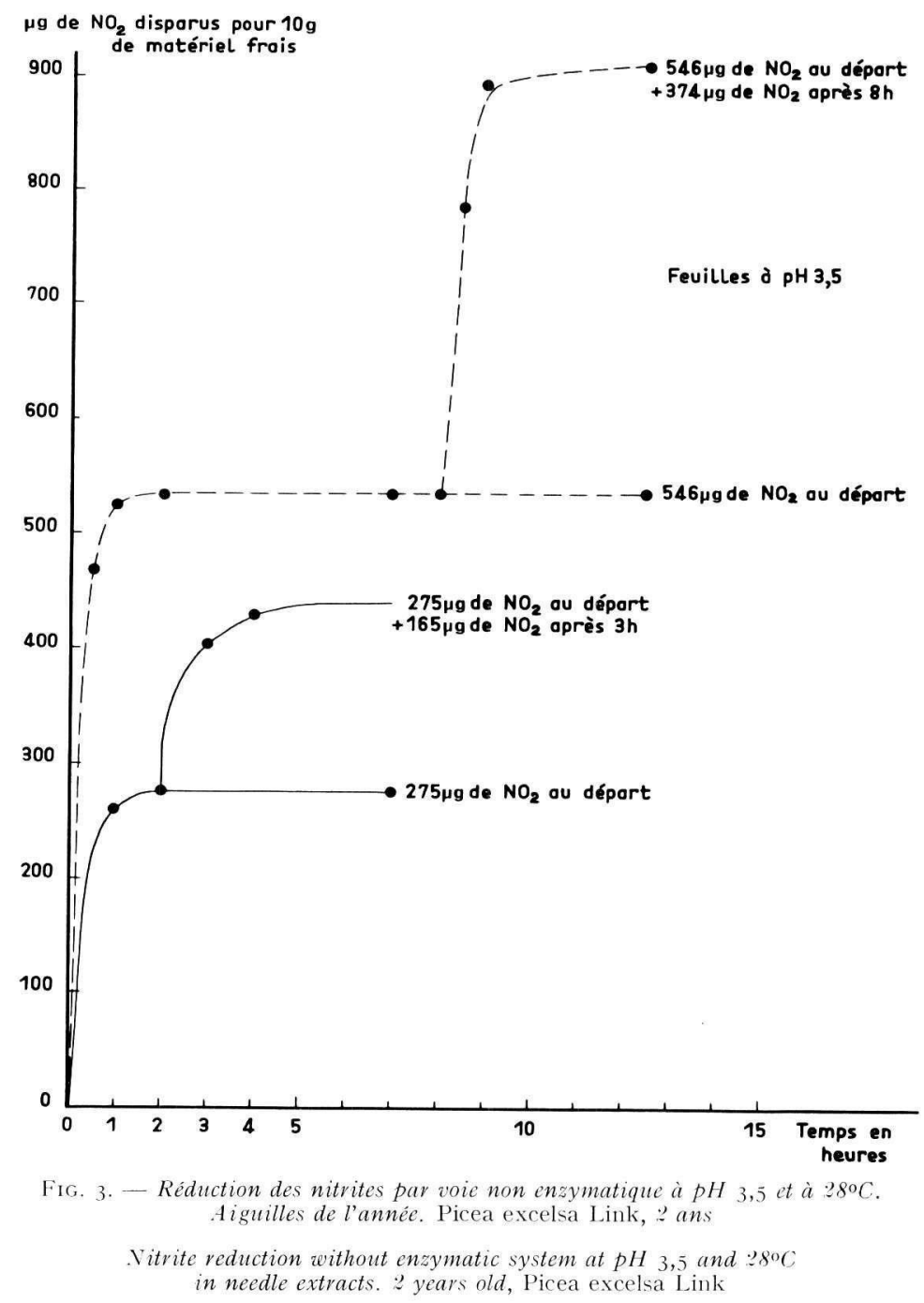

Néanmoins, dans ce dernier cas, la réduction est proportionnelle à la quantité de nitrites présents dans le milieu au départ.

Dans le cas des racines à $\mathrm{pH} 3,5$ par exemple, la réduction cesse au bout de 5 heures, bien qu'il y ait encore 30 à $5^{\circ}$ ôg de nitrites environ à réduire dans le milieu; mais suivant la quantité initiale de nitrites ( Ioo ou $200 \mu \mathrm{g}$ ), il aura été réduit 60 ou $\mathrm{I}_{50} \mu \mathrm{g}$ en 5 heures). 
Enfin, lorsque la réduction s'arrête, faute de nitrites dans le milieu elle reprend dès que 1'on ajoute à nouveau des nitrites, même au bout d'un temps assez long (8 h, fig. 3).

La lumière n'a pas d'influence sur la réduction des nitrites (fig. 4)

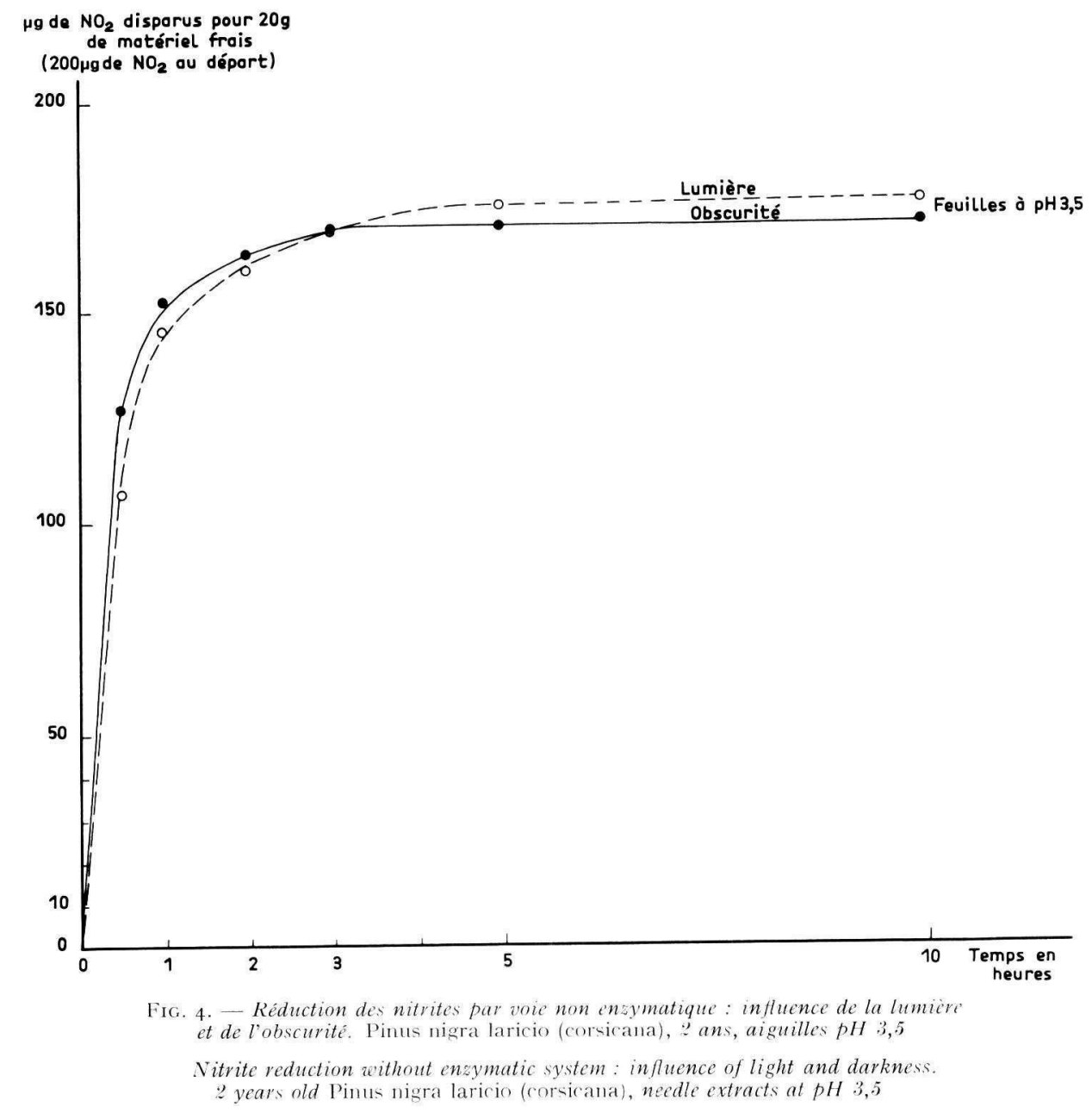

\section{5. - ACTIVITÉ DE LA NITRATE RÉDUC'TASE}

La mesure de l'activité de la nitrate réductase par dosage des nitrites apparaissant dans le milieu n'est valable que si la capacité de réduction des nitrites est nulle.

Or, nous savons que la réduction des nitrites est faible à $\mathrm{pH} 7,2$ et élevée à $\mathrm{pH} 3,5$. A pH 7,2 on détermine done surtout Pactivité de la nitrate réductase, alors qu'à $\mathrm{pH} 5$ ou 3,5 on ne détermine que la résultante de l'activité de la nitrate réductase et de la capacité des tissus à réduire les nitrites. 


\section{I. - Caractères enzymatiques de la réaction}

Lorsque le milieu d'incubation est porté à ébullition, même pendant un temps extrêmement court, il n'y a plus aucune réduction des nitrates. L'apparition des nitrites dans le milieu d'incubation semble donc bien due à l'activité de la nitrate réductase.

\section{2. - Influence du $p H$}

La figure 5 (Épicéa) montre que l'apparition de nitrites dans le milieu est nulle à $\mathrm{pH} 3,5$, faible à moyenne à $\mathrm{pH}$ 5,o et élevée à $\mathrm{pH}$ 7,2. En réalité, on peut seulement affirmer qu'à $\mathrm{pH} 7,2$ on mesure surtout l'activité de la nitrate réductase. A pH plus bas, on mesure la résultante de l'activité de la nitrate réductase et de la capacité des tissus à réduire les nitrites. On ne peut donc pas dire, au vu de ces résultats, que l'activité de la nitrate réductase soit réellement nulle à $\mathrm{pH} 3$ ou faible à $\mathrm{pH} 5$. Néanmoins, tous les auteurs qui ont travaillé sur l'enzyme purifiée sont d'accord pour affirmer que l'optimum d'activité de la nitrate réductase est bien aux environs de $\mathrm{pH} 7$ (Evans, NASON, 1953).

La figure 5 montre également que l'activité de la nitrate réductase est nettement plus importante dans les racines que dans les feuilles.

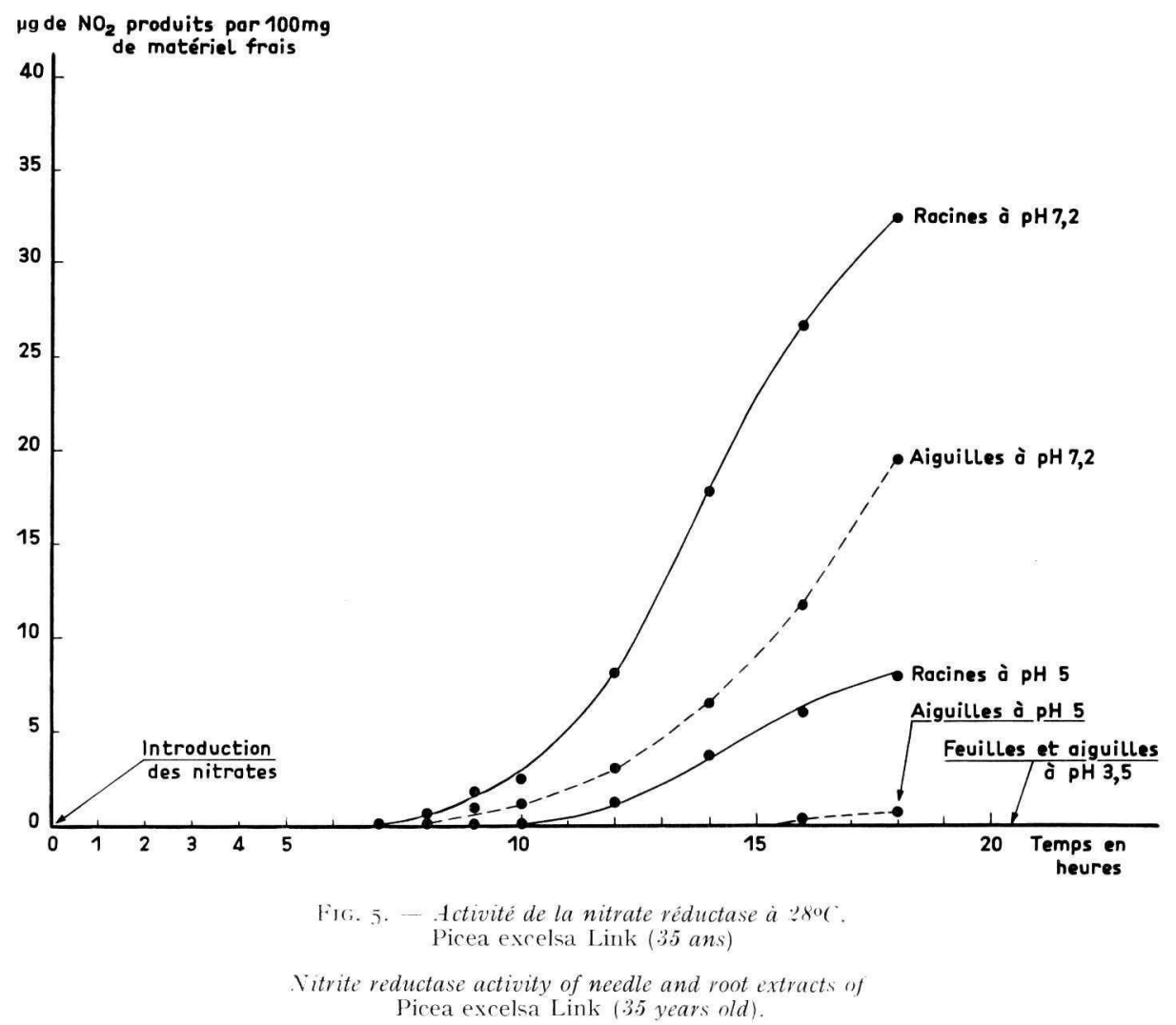




\section{3. - Temps de latence}

Nous avons observé, aussi bien sur arbres adultes que sur jeunes plants, et sur Épicéa aussi bien que sur Pin Laricio de Corse, qu'il se passait plusieurs heures avant que les nitrites n'apparaissent dans le milieu d'incubation. Ce temps de latence est de 7 à ro heures pour les tissus racinaires et plus élevé de quelques heures pour les tissus foliaires.

On ne peut attribuer l'existence de ce temps de latence à la seule capacité des tissus à réduire les nitrites, puisqu'il existe aussi bien à $\mathrm{pH} 7,2$ qu'à $\mathrm{pH} 5,0$.

Nous avons essayé d'autre part de déterminer si le développement de l'activité enzymatique était induite ou non par la présence de nitrates dans le milieu (fig. 6).

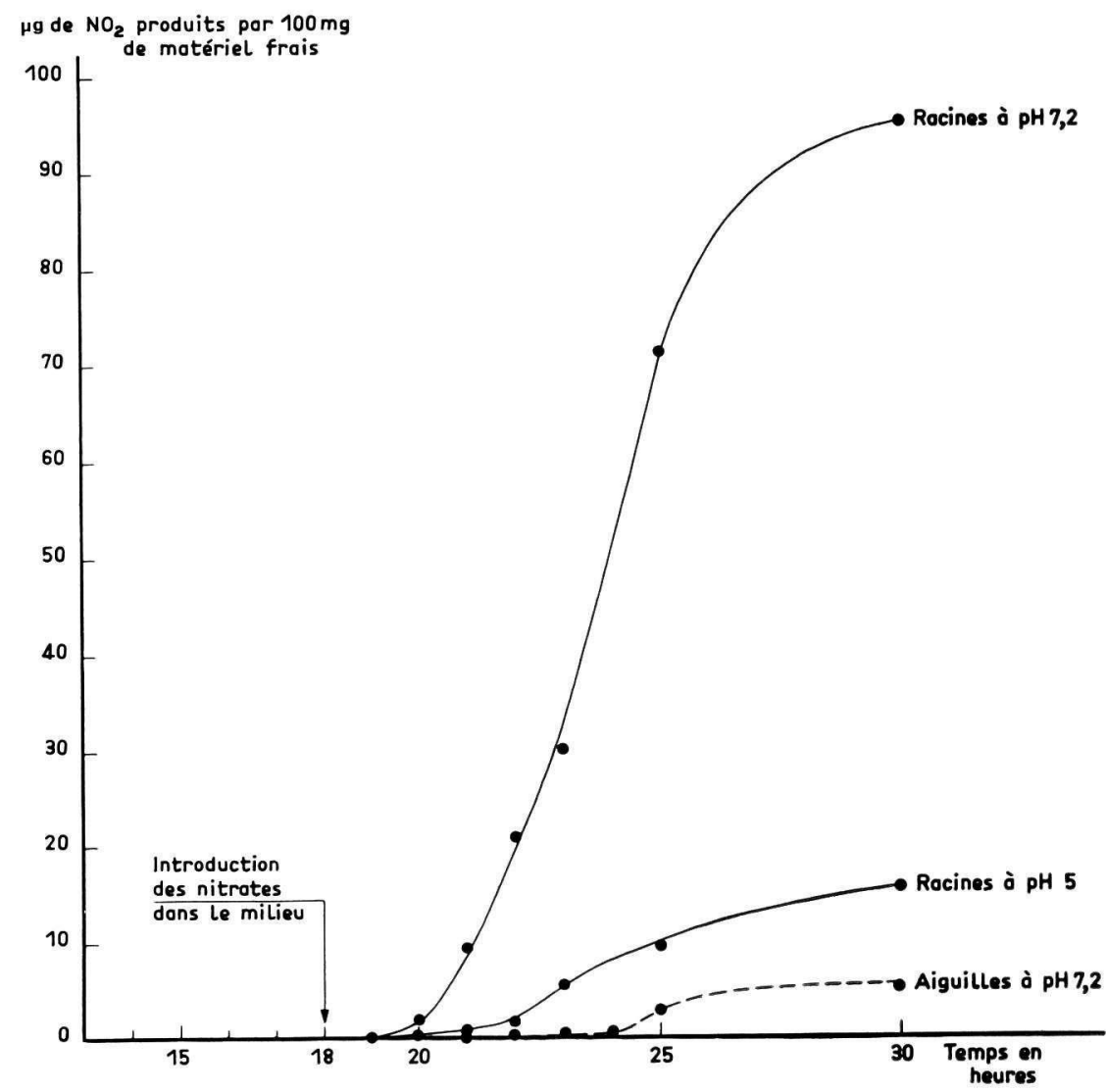

Fig. 6. - Activité de la nitrate réductase à $28^{\circ} \mathrm{C}$. Introduction des nitrates après 18 heures. Pinus nigra laricio (corsicana) 2 ans

Nitrate reductase activity of root and needle extracts of Pinus nigra laricio (corsicana). Introduction of nitrates after 18 hours

Lorsque l'on introduit les nitrates au bout de $\mathrm{I} 8$ heures, l'activité de la nitrate réductase pour des racines de Pin Laricio de Corse, à $\mathrm{pH} 7,2$ ou à $\mathrm{pH} 5$ démarre au bout de I à 2 heures, au lieu de 7 à $\&$ heures, si les nitrates sont introduits en même quantité au départ de l'expérience. 
Dans ces conditions, le temps de latence est notablement diminué. L'induction de l'activité de la nitrate réductase semble donc en partie indépendant de la présence ou de l'absence de nitrates au départ. Dans le cas des aiguilles, le temps de latence est également diminué lorsque les nitrates sont introduits après i 8 heures, mais il est encore de 6 heures.

Nous avons essayé de voir dans quelle mesure le mode de broyage du matériel végétal pouvait avoir une influence sur ce temps de latence. Cet essai a porté sur des aiguilles de Pin Laricio de Corse et comprend 3 modalités : aiguilles non broyées, aiguilles en fragments de 2 à $3 \mathrm{~mm}$, aiguilles très finement broyées. Dans cette expérience, matériel végétal et solution tampon ont été maintenus en contact jusqu'aux dosages. La figure 7 montre que seuls les traitements avec aiguilles fragmentées ou finement broyées présentent une activité enzymatique importante. Cette activité se manifeste plus tôt et avec une intensité plus grande dans le cas des aiguilles très finement broyées que dans le cas des aiguilles fragmentées.

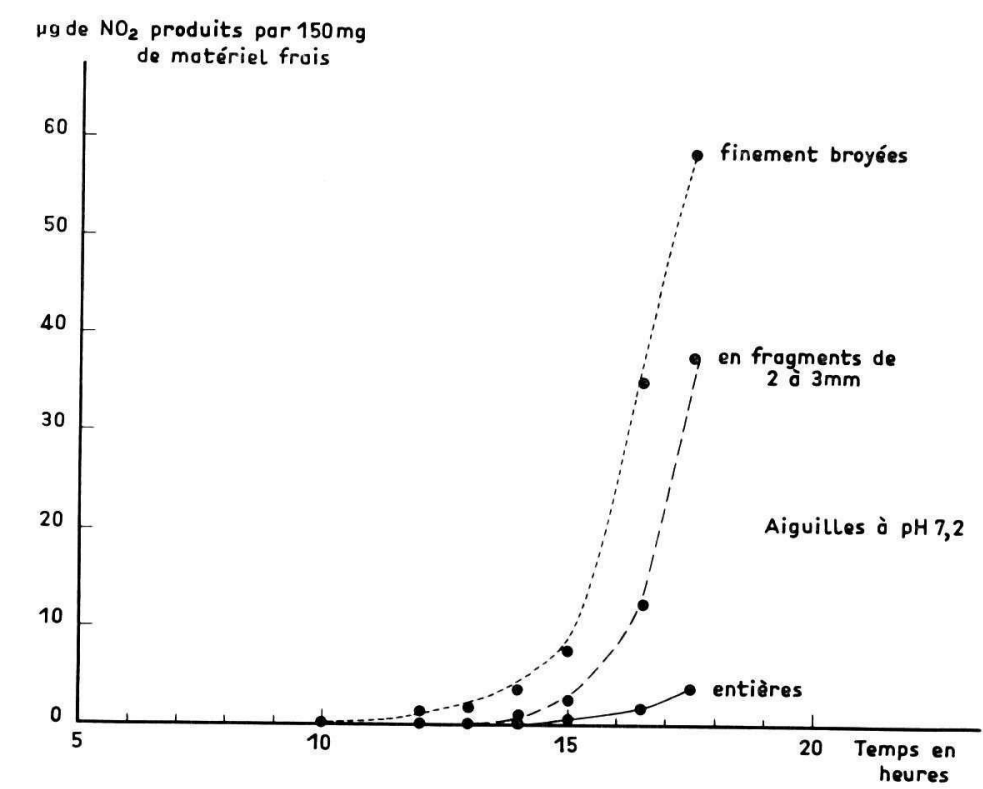

FIG. 7. - Influence du broyage sur l'activité de la nitrate réductase des aiguilles $\grave{a} 28^{\circ} \mathrm{C}$ et à $p H \quad 7,2$. Pinus nigra laricio (corsicana) 2 ans

Influence of grinding on nitrate reduction activity of needle extracts at $p H 7,2$ and $28^{\circ} \mathrm{C}$. 2 years old, Pinus nigra laricio (corsicana)

Le temps de latence est de ro heures pour les aiguilles broyées, de I 3 heures pour les aiguilles fragmentées et de I4 heures pour les aiguilles intactes.

Il semble donc que la durée du temps de latence soit fonction de l'état du matériel végétal.

Dans le cas des aiguilles non broyées, la cuticule épaisse de l'épiderme et la couche de sclérenchyme sous-épidermique freinent les échanges entre le milieu et les fragments de tissus. Lorsque le matériel végétal est finement broyé, les échanges se font plus facilement à travers les parois minces du parenchyme foliaire. 
Avec du matériel végétal à parois peu épaisses (Peuplier, Hêtre) nous avons pu vérifier que ce temps de latence n'existait pratiquement pas. Chez le Peuplier en particulier, l'activité de la nitrate réductase démarre presque immédiatement.

Il est généralement admis que ce sont les nitrates et les nitrites qui diffusent entre le milieu et les tissus et non l'enzyme. Or, nous avons pu vérifier qu'après séparation des tissus du milieu, les nitrates pouvaient encore être réduits. D'autre part, si ce milieu est passé sur un filtre millipore (diamètre des pores $0,22 \mu$ ) le filtrat n'a jamais plus la possibilité de réduire les nitrates. Il semble donc bien que, dans nos conditions d'expérience, la nitrate réductase puisse diffuser dans le milieu et qu'elle soit arrêtée en raison de son poids moléculaire élevé par un filtre millipore de $0,22 \mu$ de diamètre.

Néanmoins, si la structure des parois du matériel végétal résineux peut expliquer l'existence de ce temps de latence, cette explication ne peut être que partielle.

En effet, lorsque l'on sépare, au bout de 2 heures, le matériel végétal broyé ou fragmenté de la solution tampon, la nitrate réductase est déjà passée en grande partie dans le milieu, puisque l'on obtient une forte activité. Mais cette activité n'est obtenue qu'au bout de quelques heures. Il faut donc admettre que l'existence de ce temps de latence est aussi due, en partie, à un inhibiteur dont l'action ne dure que quelques heures. Cette inhibition temporaire semble spécifique des résineux car aucun phénomène de ce genre n'a été observé chez d'autres espèces végétales Notons cependant que WALLACE et PATE (1965) ont observé sur le pois une influence inhibitrice de la cystéine sur la nitrate réductase. D'autre part, THEOBALD et Smith (I974), sur des racines de Pinus elliotii, n'obtiennent in vitro aucune activité de la nitrate réductase. Ils attribuent ce fait à la présence d'inhibiteurs (polyphénates et tannins). Par contre, in vivo, sur fragments de racines de 8 à Io $\mathrm{mm}$, ces mêmes auteurs n'observent pas de temps de latence chez Pinus elliottii.

En résumé, le temps de latence dans l'activité de la nitrate réductase chez les aiguilles et les racines des résineux étudiés semble due à 3 causes au moins :

- Influence de la capacité des extraits de tissus à réduire les nitrites (Nous ne pouvons en effet complètement éliminer cette hypothèse, même encore à pH 7,2$)$.

- Influence de l'épaisseur des parois cellulaires : les parois épaisses de l'épiderme et des clérenchymes externes des tissus résineux sont un obstacle certain aux échanges entre milieu et fragments de tissus.

- Influence d'un inhibiteur : l'existence d'un inhibiteur à action temporaire est très probable. Cet inhibiteur, apparaissant au moment du broyage ou de la fragmentation des tissus, serait détruit dans les solutions tampons au bout de quelques heures.

\section{COMPARAISON DE L'AC'TIVITÉ DE LA NITRATE RÉDUCTASE} E'T DE LA CAPACITÉ DES TISSUS A RÉDUIRE LES NITRITES, CHEZ L'ÉPICÉA ET LE PIN NOIR, EN SOL ACIDE ET EN SOL CALCAIRE

Nous avons effectué les comparaisons sur deux couples de peuplements de Pin noir d'Autriche et d'Épicéa, de 35 ans chacun.

L'un de ces couples est situé sur un sol très calcaire de Champagne $\left(5^{\circ}\right.$ p. roo 
de calcaire total et $\mathrm{pH}$ de 8,3 en $\mathrm{A}_{1}$, azote minéral disponible essentiellement sous forme nitrique). Sur ce sol, le Pin noir se comporte normalement (production moyenne/ha/an à 50 ans : $8 \mathrm{~m}^{3}$ environ) ; sa nutrition azotée est à un niveau satisfaisant (teneur en azote total de I p. Ioo environ dans les aiguilles). Au contraire, l'Épicéa se comporte très mal sur ce type de sol. Sa production est de l'ordre de $2 \mathrm{~m}^{3} / \mathrm{ha}$ /an à $5^{\circ}$ ans ; il présente une chlorose généralisée et est carencé en azote (teneur en azote total de 0,7 p. Ioo dans les aiguilles).

L'autre couple est situé sur limons, sur un sol lessivé entièrement décarbonaté ( $\mathrm{pH}$ de $\mathrm{A}_{1}=5$, forte minéralisation de l'azote, forte disponibilité en azote nitrique et ammoniacal). Sur ce type de station, la production du Pin noir est à peine supérieure à ce qu'elle était sur la précédente (I I $\mathrm{m}^{3} / \mathrm{ha} / \mathrm{an}$ à 50 ans) ; sa nutrition azotée est à un niveau à peine supérieur( teneur en azote total de I, 2 p. Ioo dans les aiguilles). L'Épicéa, sur ce même type de sol peut exprimer tout son potentiel de croissance et a une production très élevée ( $\mathrm{I} 9 \mathrm{~m}^{3} /$ ha/an à 50 ans). Il utilise au maximum les possibilités de nutrition azotée (teneur en azote total des aiguilles de I,70 p. IOO).

Nous avons prélevé les feuilles et les racines fines de 5 arbres par peuplement. Précisons que les racines des deux espèces, aussi bien en milieu calcaire qu'en milieu acide, étaient bien pourvues en mycorhizes ectotrophes.

Les mesures de l'activité de la nitrate réductase ont été effectuées sur chaque arbre séparément, à $\mathrm{pH} 7,2$, par dosage des nitrites apparus au bout de $\mathbf{I} 8$ heures. Il est très probable que dans les tissus foliaires et racinaires, où le pH est de 3,5 à 5. l'activité de la nitrate réductase est moins importante.

TABIEAAU I

Activité de la nitrate réductase en sol acide et en sol calcaire ches l'Épicéa et le Pin noir d'Autriche (moyenne de 5 arbres. Les valeurs non significativement différentes sont jointes par un trait).

Nitrate reductase activity on acid and calcareous soils for Spruce and Austrian Pine (mean values for 5 trees) (non significative differences are shown by a line).

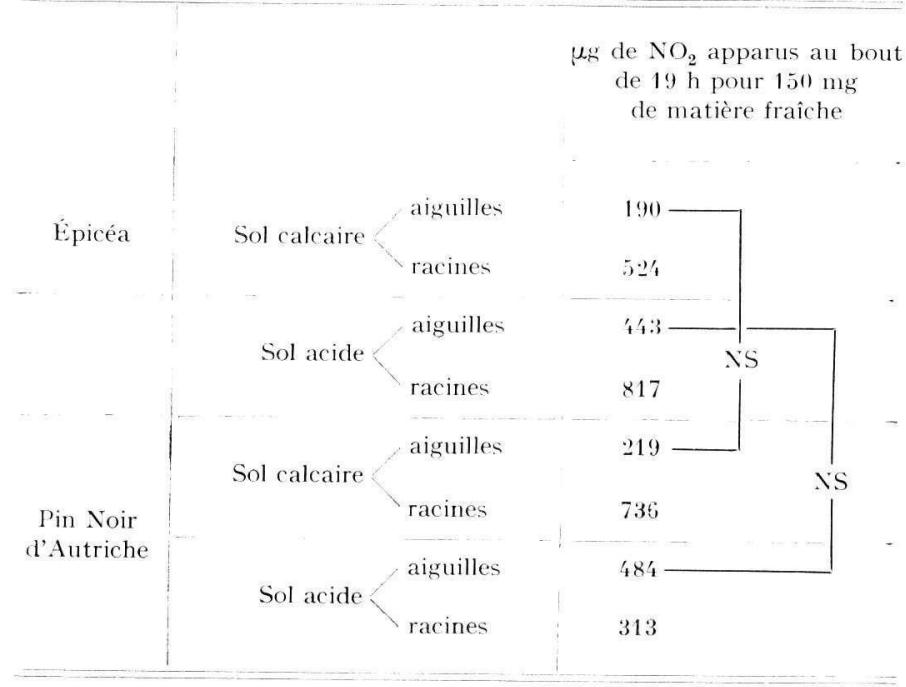


La mesure de la capacité à réduire les nitrites a été effectuée sur un mélange de tissus des 5 arbres (dosage sur feuilles et racines effectué séparément), par dosage des nitrites résiduels en fonction du temps à $\mathrm{pH} 3,5$.

Chez l'Épicéa, l'activité de la nitrate réductase est toujours beaucoup plus élevée dans les racines que dans les aiguilles. En sol acide, 1'activité est très forte dans les racines et moyenne dans les feuilles. En sol calcaire, l'activité de la nitrate réductase diminue de manière très significative; elle devient faible dans les feuilles et moyenne dans les racines.

Le comportement du Pin noir est différent. En sol acide, l'activité de la nitrate réductase est moyenne dans les feuilles et les racines et elle est un peu plus élevée dans les feuilles que dans les racines. Ein sol calcaire, l'activité de la nitrate réductase augmente dans les racines où elle devient très élevée alors que dans les aiguilles, elle diminue et devient beaucoup plus faible. Il n'y a d'ailleurs pas de différence significative entre les aiguilles de Pin noir et d'Épicéa, aussi bien en sol calcaire qu'en sol acide.

Toute la différence entre les deux espèces se situe au niveau des racines. L'activité de la nitrate réductase augmente fortement dans les racines de Pin noir lorsque l'on passe d'un sol acide à un sol calcaire (activité doublée).

Au contraire, dans les racines de l'Épicéa, l'activité de la nitrate réductase diminue lorsque l'on passe d'un sol acide à un sol calcaire.

Nous pouvons donc concevoir que l'Épicéa a des difficultés de nutrition azotée en sol calcaire. Sur ce type de station, l'azote est essentiellement sous forme nitrique. L'activité de la nitrate réductase diminuant, la réduction devient difficile, ce qui peut retentir sur tout le métabolisme de l'azote.

Le Pin noir n'a aucun problème puisque dans les racines en sol calcaire, 1'activité de la nitrate réductase est à un niveau élevé.

La production du Pin noir n'est donc pas affectée en sol calcaire, alors que celle de l'Épicéa chute considérablement. Eille chute d'autant plus que les besoins en azote de l'Épicéa sont très élevés alors que ceux du Pin noir sont, semble-t-il, plus faibles.

Il faut néanmoins faire remarquer que l'activité de la nitrate réductase dans les racines d'Épicéa en sol calcaire est loin d'être négligeable. La diminution de l'activité de cette enzyme lorsque l'on passe d'un sol acide à un sol calcaire ne peut donc être qu'une explication partielle.

Néanmoins, pour éliminer totalement l'influence de la capacité des tissus à réduire les nitrites dans la mesure de l'activité de la nitrate réductase, nous avons effectué nos déterminations à $\mathrm{pH} 7,2$. Le $\mathrm{pH}$ des tissus racinaires étant de 5 , il est très probable que l'activité de la nitrate réductase dans la réalité est beaucoup plus faible que ce que nous avons déterminé par la méthode des fragments, du moins si les sites d'activité de la nitrate réductase sont à un pH équivalent à celui de l'ensemble des tissus racinaires.

Dans cette hypothèse, l'activité de la nitrate réductase dans les racines d'Épić́a en sol calcaire ne serait plus moyenne mais faible ou très faible. En ce qui concerne la capacité des tissus à réduire les nitrites par voie non enzymatique, on constate chez le Pin noir, qu'elle est équivalente dans les racines et dans les aiguilles et qu'il n'y a pas de différence entre sol acide et sol calcaire (fig. 8).

Chez l'Épicéa, cette capacité à réduire les nitrites est toujours plus élevée que chez le Pin noir. Elle est également un peu plus élevée dans les aiguilles que dans 
les racines. Il semble enfin que ce pouvoir de réduction soit un peu plus important en sol acide. De toute façon, la capacité à réduire les nitrites reste supérieure à ce qu'elle est chez le Pin noir d'Autriche.

Le blocage de la réduction des nitrates chez 1'Épicéa en sol calcaire semble donc se faire tout au début de la chaîne, au moment de la transformation des nitrates en nitrites. Il ne semble pas y avoir de problème pour le passage des nitrites au stade suivant, ce qui ne veut pas dire d'ailleurs qu'il ne puisse pas y avoir d'autres blocages à des stades ultérieurs.

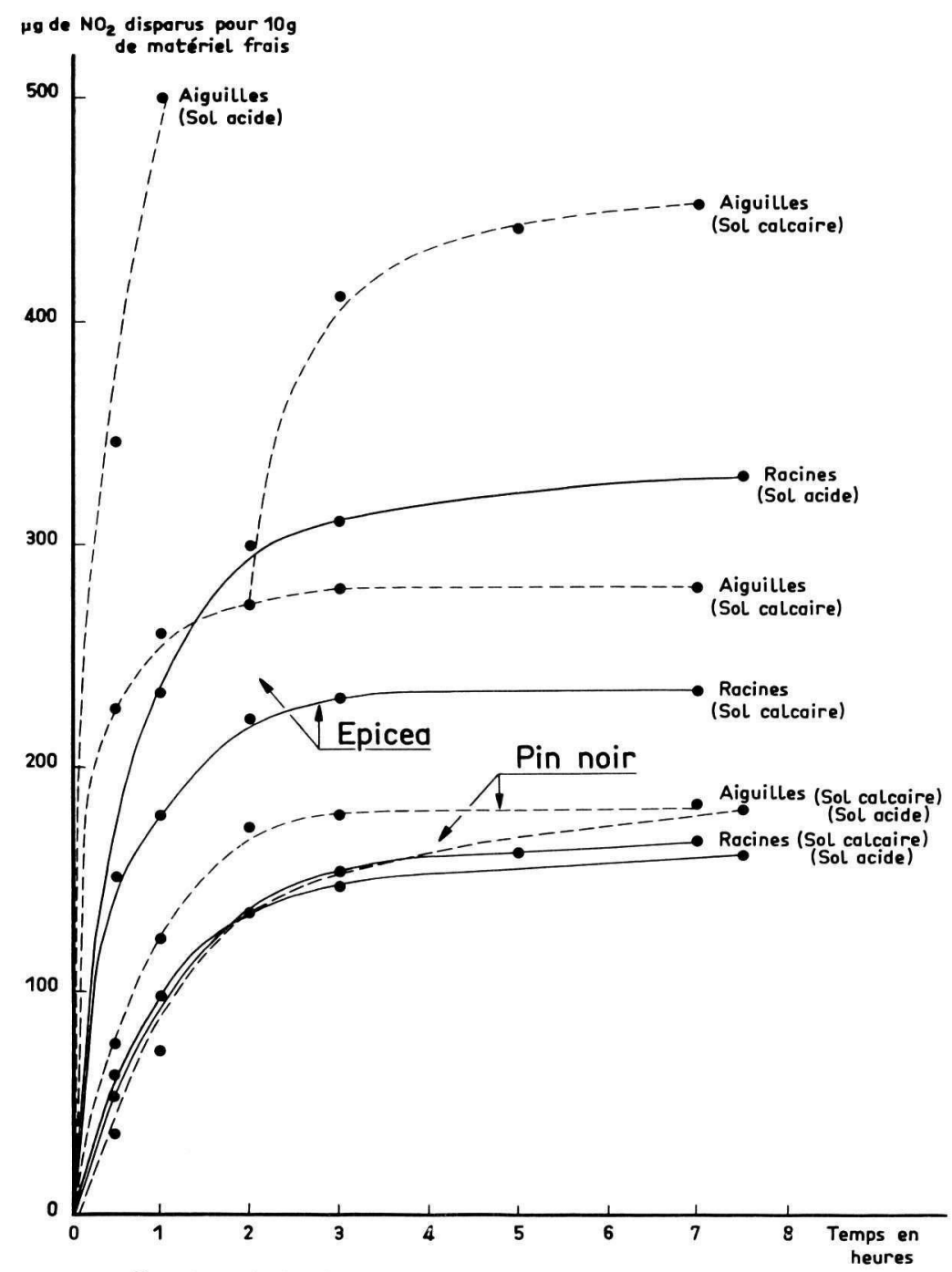

FiG. 8. - Réduction des nitrites par voie non enzymatique. Comparaison Épicéa (Picea excelsa Link) et Pin noir d'A utriche (Pinus nigra nigricans austriaca) en sol acide et en sol calcaire (aiguilles et racines)

Reduction of nitrite without enzyme system in needle and root extracts of Picea excelsa Link and Pinus nigra nigricans. Influence of soil 


\section{CONCLUSIONS}

Lorsque l'on détermine la quantité de nitrites apparus dans un milieu contenant des fragments de tissus végétaux, après introduction de nitrates, on mesure la résultante de deux phénomènes : l'activité de la nitrate réductase qui produit les nitrites et la capacité des tissus à réduire les nitrites en composés moins oxydés, par voie non enzymatique. Nous avons pu dissocier ces deux phénomènes sans pratiquer de séparation moléculaire, en agissant uniquement sur le $\mathrm{pH}$ du milieu.

Nous avons retenu les $\mathrm{pH}$ de 3,5 pour étudier la capacité à réduire les nitrites par voie non enzymatique, et de 7,2 pour étudier l'activité de la nitrate réductase. A pH 3,5, l'activité de la nitrate réductase est très faible; elle est à son optimum à $\mathrm{pH} 7,2$. Inversement, la capacité des tissus à réduire les nitrites est très élevée à $\mathrm{pH} 3,5$ et faible à $\mathrm{pH} 7,2$. Nous avons complété notre étude par une mesure globale de l'activité de la nitrate réductase et de l'aptitude des tissus à réduire les nitrites par voie non enzymatique à $\mathrm{pH} 5$ ( $\mathrm{pH}$ des tissus racinaires).

L'aptitude à réduire les nitrites n'est pas influencée par la lumière et devient rapidement très importante dès l'introduction de nitrites dans le milieu, puis cette activité baisse et s'annule tout en laissant dans le milieu une quantité résiduelle de nitrites variable suivant le $\mathrm{pH}$ utilisé ou la nature des tissus étudiés.

L'aptitude à réduire les nitrites est, chez l'Épicéa, un peut plus élevée dans les tissus foliaires que dans les tissus racinaires; chez le Pin noir cette activité semble équivalente dans les deux tissus.

Nous avons mis en évidence une période de latence précédant le démarrage de l'activité de la nitrate réductase. Ce temps de latence est compris entre 7 et io heures dans les tissus racinaires et supérieur à Io h dans les tissus foliaires des résineux. Nous savons que ce temps de latence est faible ou n'existe pas chez les végétaux cultivés et les essences feuillues.

La durée du temps de latence est fonction du degré de broyage des tissus. La structure des parois des tissus de résineux ne peut cependant que partiellement expliquer l'existence de ce temps de latence. Nous devons admettre l'existence d'un inhibiteur enzymatique, actif pendant les quelques heures qui suivent le broyage ou la fragmentation des tissus.

Chez tous les résineux que nous avons étudiés, l'activité de la nitrate réductase à $\mathrm{pH}$ 7,2 est beaucoup plus élevée dans les racines que dans les aiguilles. La comparaison de l'activité de l'enzyme chez 1'Épicéa (calcifuge) et le Pin noir (tolérant ou calcaire), a montré un comportement très différent des deux espèces au niveau des racines. Chez le Pin noir, lorsque 1'on passe d'un sol acide à un sol calcaire, on constate une augmentation de l'activité de la nitrate réductase dans les racines. Chez l'Épicéa, il se produit 1'effet inverse. Au niveau des aiguilles, par contre, nous n'avons pas constaté de différence de comportement entre les 2 espèces.

Ces observations permettent d'expliquer, du moins partiellement, les différences importantes de la nutrition azotée qui existent chez 1'Épicéa en sol calcaire. L'azote nitrique est pratiquement la seule forme d'azote disponible. La faible activité de la nitrate réductase sur ce type de sol entraîne une réduction difficile dans les racines. 
Le métaDolisme de l'azote est donc de ce fait perturbé chez l'Épicéa qui ne peut ainsi faire face à ses besoins en azote.

Par contre, l'activité de la nitrate réductase est élevée dans les racines de Pin noir en sol calcaire. Le Pin noir peut donc s'approvisionner en azote sans difficulté et ne présente pas, de ce fait, de déficience en azote en sol calcaire, ni de chlorose.

Nous avons relevé chez 1'Épicéa en sol calcaire des difficultés au niveau de la réduction des nitrates, c'est-à-dire dès la première étape du métabolisme de l'azote. Il n'est pas impossible que d'autres difficultés existent plus loin dans la chaine de transformation de l'azote.

Reç pour publication en décembre 1975

\title{
SUMMARY
}

OBSERVATIONS ON NITRATE REDUC'TASE EFFICIENCY

AND ON NON ENZYMATIC NITRITE REDUCTION IN SOME CONIFEROUS TREES

Nitrate reduction activity in tissues of coniferous trees is high at $\mathrm{pH} 7.2$ and very low at $\mathrm{pH} 3,5$. Nitrate reductase activity is more important in roots than in needles. It begins only several hours (7-IOh) after extraction. The length of this period of inactivity depends of the grinding degree of tissues, and is likely to depend of an enzymatic inhibition.

Nitrites reduction seems to be non enzymatic : after boiling, tissues are still able to reduce nitrites. This ability is high at $\mathrm{pH} 3.5$ and very low at $\mathrm{pH} 7.2$. It seems to be higher in foliar tissues that in root tissues.

We compared this system of nitrates and nitrites reduction for two species (Picea excelsa Link and Pimus nigra nigricans) on two soils (on acid soil and a calcareous soil).

In roots tissues of Pinus nigra nigricans, nitrate reductase activity is higher on calcareous soil, than on acid soil. It is exactly the contrary for Picea cxcelsa Link. Theses observations can partially explain the difficulties of nitrogen nutrition of Picea excelsa Link on calcareous soils.

\section{ZUSAMMENFASSUNG}

\author{
BEOBACHTUNGEN ÜBER DIE WIRKSAMKEIT DER NITRAT-REDUKTASE \\ UND DIE MÖGLICHKEIT EINER NICHT ENZYMATISCHEN NITRITREDUKTION \\ BEI EINIGEN NADELBÄUMEN
}

Die Aktivität der Nitrat-Reduktase in Gewebe von Nadelbäumen ist bei einem pH-Wert um 7,2 sehr hoch, bei cinem $\mathrm{pH}$-Wert von 3,5 jedoch sehr gering. Sie ist in den Wurzeln bedeutend höher als in den Nadeln. Es zeigt sich eine Latenzperiode von 7 bis ro Stunden, bevor die NitratReduktase ihre Aktivität beginnt. Diese Latenzperiode ist von der Aufbereitung der Gewebe abhängig und dürfte auf das Vorhandensein eines Hemmstoffes zurückzuführen sein.

Die Reduktion der Nitrite scheint nicht durch Enzyme zu erfolgen. Das Reduktionsvermögen der Gewebe ist sehr hoch bei $\mathrm{pH} 3,5$ und praktisch null bei $\mathrm{pH}$ 7,2; es scheint bei Nadel höher als bei Wurzel zu sein.

Die Untersuchungen wurden an Fichte (Picea abies karst.) und Schwarzkiefer (Pinus nigra nigricans) von bodensauren und kalkreichen Standorten durchgeführt.

Die Aktivität der Nitrat-Reduktase im Wurzelgewebe der Schwarzkiefer ist auf kalkreichen Standorten höher als auf bodensauren Standorten. Bei der Fichte konnte das Gegenteil beobachtet werden. Dies erklärt zum Teil, die bei der Fichte auf Kalkstandorten festgestellten Schwierigkeiten der Stickstoffernährung. 


\section{RÉFÉRENCES BIBLIOGRAPHIQUES}

BAR-AKIVA A., I 968 . Investigation into new methods for assessing nutrient status (deficiences and excesses) in (itrus trees and other plants. Bet Dagan Israël final report of Research.

Bak-Akiva A., Sagiv J., Leshem J., 1970, Nitrate Reductase activity as an indicator for assesing the Nitrogen Requirement of grass crops. J. Sci. Fd. Agric., 21, August, 405-407.

Bar-Akiva A., Sternbadm, 1965. Possible uso of nitrate reductase activity of leaves as a mesure on the nitrogen requirement of Citrus trees. Plant Coll Physiol., (6), 575-577.

Bar-Akiva A., Sternbaum, 1965. Nitrate reduction in Citrus trees leaves. Plant and somi, XXIII, (I), I4I-I 44 .

Barnes, Folkhard, 1951. Détermination of nitrites. Analyst, 76, p. 599.

Bafleks L., FLescher D). HAGEMan R. H., rg64. Studies of the pyridine nucleotide specificity of nitrate reductase in higher plants and its relationships to Sulfhydryl level. Biochim. hiophys. Acta, $89,453-464$.

13.ANC D., 1962. Absorption et utilisation comparée des nitrates par différentes souches de graminces fourragères. CR, Acad. Agric. France, I53-160.

Blondel A. M., 1971. Mesure de l'activité de la nitrate réductase. Esiál de mise au point d’un tesi rapide par la méthode des fragments. I)FA, Antibes, France.

Candela M. L., Fischer E. G., Hewitt E. J., i957. Molybdenum as a plant nutrient. Some factors, affecting the activity of nitrate reductase in Cauliflower plants with different nitrogen sources and molybdenum levels in sand culture. Plant Physiol., 32, 280-287.

Champigny M. L., 1963. Sur l'activité et l'induction de la nitrate réductase dans les plantules de mais. Physiol. vég., 1 (2), 1 39-169.

Clement A., 1972. Étude de l'équilibre acido-basique de l'lipicéa sur sol très carbonaté, carhonuté et décarbonaté. Thèse de spécialité. Nancy, France.

Chiment A., 1974. Étude de la nutrition minérale de Picea excelsa Link sur sol calcaire et sur sol décarbonaté, incidence de la nutrition sur le métabolisme des anions minéraux et organiques. Ann. Sci. forest., (4), I $89-205$.

D)chavfolr P., ig6o. Notes sur l'origine de la carence de certains conifères sur sol calcaire. Bull. ioc. Bot. France, 10\%, 8-12.

1)rchaufour P., Bonneau M., I962. Étude expérimentale de l'influence du calcaire sur la nutrition et la croissance d'un résineux acidiphile. Annales de l’E.X.E.F. et de la Station de Recherches et Expériences tome XIX, fasc. 3 .

D) кstra G. F., I974. Nitrate reductase activity and protein concentration of two Poplars clones. Plant Physiol., 53, 632-634.

Evans H. J., Nason A., I 953. Pyridine nucleotide-nitrate reductase from extracts of higher plantPlant Physiol., 28, 233-54.

(iARbase J., Le TACon F., 1974. Etude expérimentale du comportement de diverses essences résineuses et de deux clônes de peupliers vis-à-vis de pH et de la teneur en calcaire du sol. Req. forest. fr., (5).

(irasmanis V. O., Nicholas D. J. I), ig67. A nitrate reductase from apple roots. Phytochemistry, 6, $217-218$.

Hageman R. H., Flescher 1), I960. Nitrate reductase activity in corn seedlings as affected by light and nitrate content of nutrient media. Plant Physiol., 35, 7oo-7o8.

Hewitt E. J., Nicholas D. J. D., I967. In Modern Methods of Plant Analysis, Edited by : Linskixs H. F., Sanual B. D., Tracey M. W., V'il, p. 67. Springer Verlag-Berlin Göttingen, Heidelberg.

Hewitt E. J., Hucklessy B. P., Betts G. F., 1968. Vitrite and hydroxylamine in inorganic nitrogen metabolism with reference principally to higher plants. Note ronéotypée. Long Ashton Research Station, Iniversity of Bristol.

Jaworski E. G., r971. Nitrate reductase assay in intacts plant tissues. Biochem. Biophys. Res. Comm., 43, (6), I274-1279.

KLepper L., Hageman R. H., ig69. The occurrence of nitrate reductase in Apple leaves. Plant Physiol., 44, IIO-II4.

Kramer P. J., Kozlowski T. T., i960. Physiology of trees. McGraw-Hill Co, Inc. New York.

Krupa S., Fontana A., Palenzona M., 1973. Studies on the nitrogen metabolism in ectonycorrhizae. I. Status of free and bound amino-acids in mycorrhizal and non mycorrhizal root systems of Pinus nigra and Coryllus avellana. Physiol. Plant., 28 (I), I-6.

Le Tacon F., Millier C., r970. Influence des conditions de nutrition minérale sur la croissance de l'Épicéa commun sur les plateaux calcaires de l'Est de la France. Ann. Sci. forest., 27 (4), 335-355.

Le Tacon F., Millier C., i97o. La nutrition minérale de l'Épicéa commun (Picea excelsa Link) en sols carbonatés et en sols décarbonatés. Essai sur le comportement du calcium et du manganèse. Ann. sci. forest., (I), I-24. 
Muldek li. G., Boxmar, Van VEen W. L., 1959. The effect of molybdenum and nitrogen deficiencies on nitrate reduction in plant tissues. Plant and Soil, X, (4), 335-355.

Nicholas D, J. D., Scawix J. H., 1956. A phosphate requirement for nitrate reductase from Neurospora crassa. Nature, 178, 1474-1475.

Pate J. S., Wallace W., I964. Movement of assimilated nitrogen from the root system of the field pea (Pisum ariense L.). Ann. Bot., 28 (1o9), 83-99.

RANDALl P. J., 1969. Changes in nitrate and nitrate reductase levels on restoration of molybdenum to molybdenum deficient plants. Aust. J. agr. Res., 20, 635-642.

Stevens I), L., OAKs Ann, I973. The influence of nitrate on the induction of nitrate reductase in maize roots. Can. J. Bot., 51, 1255-1258.

Streeter J. G., Bosler M. E., 1972. Comparaison of in vitro and in vivo assays for nitrate reductase in Soybean leaves. Plant Physiol., 49, 448-450.

Theовадd W. F., Sмiтн W. H., i974. Nitrate production in two forest soils and nitrate reduction in pine soil. Sci. Soc. Ann. Proc., 38, 668-672.

Wallace: W., Pate J. S., I965. Nitrate reductase in the field pea (Pisum arvense I..). Ann. Bot. 29 (다), 655-67г. 